\title{
Formation des mots : le point de vue diachronique
}

\author{
Rainer, Franz \\ Université des sciences économiques de Vienne \\ franz.rainer@wu-wien.ac.at
}

\section{Où en sont les études...}

Même si le nombre de participants à notre section semble prouver le contraire, on peut quand même constater que la formation des mots du français a connu un certain renouveau depuis deux décennies. Renouveau qui, en France, a trouvé son impulsion surtout, au début, grâce à l'activité infatigable de Danielle Corbin, décédée prématurément, et de son groupe de recherche à Lille III, mais qui entre temps a atteint d'autres villes universitaires comme Paris ou Toulouse, pour ne nommer que les plus importantes.

Ce renouveau toutefois a concerné presque exclusivement l'étude de la formation des mots dans le français actuel. La Bibliographie Linguistischer Literatur (Francfort : Klostermann 1976ss.) contient, bon an mal an, une vingtaine de travaux dédiés à la formation des mots du français, dont le nombre de ceux qui sont écrits dans une perspective diachronique ne dépasse que rarement 10 pour cent, et il y a des années où aucun travail diachronique n'est recensé. Il n'en a pas toujours été ainsi. Dans la bibliographie raisonnée de Schpak-Dolt (2003), par exemple, qui inventorie 665 travaux sur la morphologie française flexion incluse - publiés entre 1875 et 1950, l'approche diachronique est encore très largement prépondérante. Il n'est pas difficile d'identifier la raison de cette inversion de tendance spectaculaire en faveur de la synchronie : le structuralisme, le générativisme et, plus généralement, 68, du moins dans les pays de langue allemande, ont porté un coup dur à l'étude de l'ancien français.

Une autre différence voyante entre les deux bibliographies concerne le métalangage : tandis que la moitié des contributions recensées par Schpak-Dolt sont écrites en allemand, plus de $90 \%$ des travaux récents sont écrits en français. Le fait qu'une portion si importante des travaux de l'époque diachronique, dont la consultation est toujours recommendable, ait été écrite en allemand constitue certainement un obstacle ultérieur pour les francophones qui voudraient se dédier à l'étude de l'histoire de la formation des mots en français.

\section{Les manuels}

Le français ne dispose pas de manuels à jour sur la formations des mots. C'est sans doute la lacune la plus douloureuse de notre domaine de recherche. Les manuels récents sont tous de type pédagogique, et ne permettent donc nullement au lecteur de se faire une image fidèle de l'état de la recherche, ni de la bibliographie existante. L'intention pédagogique de ces manuels comporte aussi une approche presque exclusivement synchronique.

Pour la diachronie, il faut toujours recourir à Nyrop (1908) et, si on lit l'allemand, à Meyer-Lübke (1921). Ces deux manuels, très méritoires en leur temps, ne reflètent plus, sur beaucoup de points, l'état actuel de la recherche. De l'élégant manuel de Meyer-Lübke, il existe bien une édition mise à jour en 1966, par les soins de son élève J. M. Piel, mais celui-ci, malheureusement, ne s'est pas montré à la hauteur de la tâche (v. les comptes-rendus substantiels de Höfler 1967, Bork 1968, Jänicke 1969). ${ }^{1}$

La situation n'est pas plus réjouissante en ce qui concerne la description synchronique d'états de langue passés. Les grammaires de l'ancien français n'incluent pas, normalement, de chapitre sur la formation des mots. $^{2}$ Buridant $(2000: 14)$ dit que des "contraintes éditoriales" l'auraient empêché de retenir, dans sa Grammaire nouvelle de l'ancien français (Paris : Sedes 2000) un dernier chapitre consacré à la morphologie dérivationnelle. Entre temps, on peut du moins consulter ses Prolégomènes (Buridant 2000), qui font le tour des travaux existants et des multiples problèmes méthodologiques liés à un tel projet, 
notamment la prise en compte des dimensions diatopique (dialectes, scriptce) et diaphasique (genres textuels).

\section{Les sources}

Si les manuels de Nyrop et de Meyer-Lübke sont aujourd'hui obsolètes, ce n'est pas tellement pour des raisons méthodologiques - la conception analogique de la formations des mots qui est à leur base est toujours d'actualité (v. l'exposé de G. Dal à cette table ronde) -, mais parce que le grand essor de la lexicographie historique du français a eu lieu après leur publication.

Dans ce contexte, il faut, bien sûr, mentionner en premier lieu le Französisches Etymologisches Wörterbuch (Bâle : Zbinden 1928ss.), l'œuvre monumentale initiée par W. von Wartburg et poursuivie aujourd'hui sous la direction de J.-P. Chauveau, dont la consultation toutefois n'est pas aisée ${ }^{3}$ : c'est encore une fois l'allemand qui constitue pour beaucoup de non-germanophones l'obstacle le plus important pour son usage dans l'étude diachronique de la formation des mots, mais rappelons que les gloses du moins sont données en français. Pour l'ancien français, à part le Dictionnaire de l'ancienne langue française (10 voll., Paris : Vieweg 1881-1902) de F. Godefroy, signalons l'achèvement, sous l'égide de H. H. Christmann, de l'Altfranzösisches Wörterbuch (11 voll., Wiesbaden : Steiner 1925-2002) d'A. Tobler et E. Lommatzsch. L'étude de la suffixation est grandement facilitée par le Dictionnaire inverse de l'ancien français de D. G. Walker (Ottawa 1982), basé sur le Tobler/Lommatzsch. ${ }^{4}$ Le Dictionnaire étymologique de l'ancien français (Tübingen : Niemeyer 1974ss.), commencé par K. Baldinger, ne couvre jusqu'à maintenant que les lettres H à JOR. Concernant l'époque qui va de 1330 à 1500, le Dictionnaire du Moyen Français est, lui aussi, désormais achevé et disponible sur internet. ${ }^{5}$ Pour le $\mathrm{XVI}^{\mathrm{e}}$, on dispose depuis longtemps du Dictionnaire de la langue française du seizième siècle (7 voll., Paris: Champion 1925-67) d'E. Huguet. Et pour les Temps Modernes, le Trésor de la Langue Française informatisé $e^{6}$ met à disposition une masse d'informations qui attend, elle aussi, d'être mise à profit dans des études monographiques sur l'histoire de la formation des mots. Les notes étymologiques de ce dictionnaire, d'ailleurs, sont en train d'être mises à jour dans le cadre du projet TLF-étym. ${ }^{7}$ À cette série extraordinaire de dictionnaires, s'ajoute un nombre important de textes accessibles désormais électroniquement, comme ceux de Frantext ${ }^{8}$ ou de Blum (2001).

\section{Directions actuelles de la recherche et perspectives}

Ces dictionnaires sont sans doute bien connus des participants de notre section. Si je les ai énumérés de nouveau ici, c'est pour donner plus de force au paradoxe suivant : comment se fait-il que la langue qui dispose des meilleurs dictionnaires historiques et étymologiques du monde ${ }^{9}$ affiche un score si maigre en ce qui concerne l'étude diachronique de la formation des mots? Les études monographiques d'envergure qui exploitent à fond ces trésors lexicographiques se comptent en effet sur les doigts de la main. Dans ce qui suit, je mentionnerai brièvement quelques travaux récents qui s'inscrivent dans la perspective diachronique (ou la description synchronique d'un état de langue passé), en me limitant essentiellement aux vingt dernières années.

Comme étude exemplaire qui a mis à profit les richesses du FEW, à défaut d'un exemple plus récent, je signalerai un travail qui remonte déjà aux années 70, Lindemann (1977), dédié au remplacement de -eresse par-euse et -trice au $\mathrm{XVI}^{\mathrm{e}}$ siècle suite à la décadence des noms d'agents en -ere. Cette thèse de doctorat, qui se base sur un dépouillement exhaustif du FEW, suit une approche onomasiologique : elle étudie de près une catégorie dérivationnelle, en l'occurrence la féminisation des noms de personnes, et essaie de dégager les restrictions des suffixes en lice et les causes de la déchéance de l'un et du triomphe des autres. Cette étude constitue toujours un modèle à suivre.

Les suffixes tombés en disgrâce dans la langue standard, d'ailleurs, se sont souvent conservés dans les dialectes. C'était aussi le cas de -eresse (v. Lindemann 1977 : 46ss. et Lechanteur 1999 ; sur -eur, Lachance 1988). La prise en compte des dialectes est souvent fondamentale pour la reconstruction des états de langue anciens. ${ }^{10}$ Ce sont les dialectes de la langue d'oïl, p. ex., qui m'ont fourni dans Rainer 
(2005b) la solution au problème épineux de la relation entre noms d'agents et noms d'instruments en -eur : tandis que l'usage instrumental a traditionnellement (v. Baldinger 1972) été considéré comme le résultat d'une extension métaphorique à partir du sens agentif, l'absence de telles extensions dans les dialectes nous fournit la preuve qu'il n'y a jamais eu d'extension métaphorique en latin vulgaire ou en protoroman. La "polysémie" agent/instrument est donc le résultat d'autres mécanismes (emprunts, ellipses). Les dialectes sont aussi cruciaux quand il s'agit de faire l'histoire des suffixes populaires et argotiques (v., p. ex., Buchwald 1992, Baldinger 1995 sur -zingue, 1997 sur -uche, Rézeau 1997-98 et Roché 2002, 2008 sur-ouille).

L'approche sémasiologique domine dans l'étude récente de M. Roché (v. Roché 2006) sur le suffixe -ier, fortement polysémique, en ancien français. Cette étude, qui séduit par la finesse et le réalisme de son analyse sémantique, peut aussi servir d'antidote à la pratique, portée à des extrêmes invraisemblables dans Corbin/Corbin (1991) mais aussi omniprésente dans les travaux d'inspiration guillaumienne, de vouloir à tout coût réduire la polysémie des suffixes à un signifié unitaire à un niveau "abstrait". Je ne veux certes pas plaider pour une multiplication prceter necessitatem des entités, mais là où les sens concrets observables dans les textes ne sont plus dérivables automatiquement et naturellement par des inférences pragmatiques à partir d'un sens abstrait (Wortbildungsbedeutung, en allemand), il vaut mieux opter pour la polysémie. Les locuteurs, contrairement à certains linguistes, n'ont pas peur de la polysémie, au contraire : une étude attentive de l'évolution sémantique des affixes à travers les temps montre souvent une tendance inexorable à la fragmentation sémantique (v. Rainer 2003, et plus généralement sur les mécanismes du changement sémantique dans la formation des mots, Rainer 2005a).

Dans des cas concrets, il est souvent difficile de décider combien de sens exactement on peut ou doit attribuer à un affixe particulier. Ce problème est au centre de Martin (2006), qui se base sur les matériaux du DMF, qu'il a lui-même dirigé, et propose d'en distinguer trois sens au lieu d'un seul ou de deux, comme le voulait la tradition, à savoir 'éloignement', 'inversion' et 'extraction', sens qui sont issus respectivement des préfixes latins $d e$-, dis- et $e x$ - (renforcé par $d e-/ d e s-) .{ }^{11}$ À part les préfixes, l'ancienne langue se servait aussi couramment de "préverbes" pour moduler le sens des verbes (v. Buridant 1995, Turcan 2001), usage qui, en français moderne, s'est atrophié. Mais il existe aussi, en français, des constructions comparables du type aller dehors, tomber dessus, etc., constructions qui se trouvent à michemin entre syntaxe et formation des mots ${ }^{12}$ et qui ont attiré l'attention dernièrement des spécialistes d'un grand nombre de langues, dont aussi une langue sœur du français, l'italien (v. Cini à paraître). Il serait d'un grand intérêt d'approfondir les études du phénomène en français, tant d'un point de vue typologique que de celui de la reconstruction de l'évolution du latin aux langues romanes.

La perspective romane est essentielle pour bien apprécier certains phénomènes de la formation des mots en français, surtout dans une perspective diachronique. Je me limiterai ici à attirer l'attention sur Bork (1990), qui rejette l'analyse traditionnelle selon laquelle les composés VN seraient le résultat de la réanalyse de phrases à l'impératif et propose d'établir une filiation directe entre ce type de composés et les composés latins du type Verticordia 'tourne-cœur(s)' (surnom de Vénus). Les spécialistes ont été unanimes à louer les qualités philologiques de ce livre, mais sont restés divisés sur la substance (pour : Lindner 2003 : 131, Lüdtke 2005 : 274-279 ; contre : Löfstedt 1994, Gather 2001 : 192-209).

La langue française écrite, comme on sait, a commencé à intégrer des mots latins dès les tout premiers textes. L'étude diachronique de la formation des mots se voit donc pratiquement toujours devant la tâche de déterminer l'étendue de l'influence latine dans l'évolution d'un affixe ou type compositif particulier. La plupart du temps, cette influence est évidente pour des raisons formelles, mais il existe aussi des cas plus subtils. Buridant (2000 : 16-17), p. ex., fournit des observations intéressantes sur la concurrence, en ancien français, entre -ere/-eor et les constructions périphrastiques du type cil qui + verbe : avant la relatinisation massive en moyen français, ces dernières étaient utilisées fréquemment pour rendre des noms d'agents latins en -tor quand un dérivé français correspondant faisait défaut. Un problème d'un autre ordre a été traité par Thibault (1989 : 85-110), à savoir les techniques adoptées par le français dans l'adaptation des mots latins en -uus (-ceus, -eus et -ius, d'ailleurs, ont causé le même genre de problèmes ; v. hebraus $>$ hébreu, phariscus > pharisien, pygmous > pygmée, etc.). 
L'influence du latin a connu des hauts et des bas, mais elle n'a jamais cessé complètement. Une époque de latinisation intense a été celle de l'essor des sciences, qui commence au XVII siècle et dure jusqu'au XIX quand le latin est abandonné définitivement comme langue scientifique dans la plupart des pays européens. À cette époque, ce n'est plus le latin classique qui fournit les modèles, mais le latin scientifique. Les lexicographes et morphologues continuent à sous-estimer fortement le rôle du latin à cette époque, pour la simple raison qu'il n'y a pas de dictionnaire du latin scientifique qu'ils pourraient consulter, comme il y en a pour le latin du Moyen Âge et, depuis quelque temps, aussi pour la Renaissance (v. Hoven 1994). Dans l'étude diachronique de la formation des mots dans certaines langues de spécialités particulièrement latinisantes, comme celles de la médecine ou de l'histoire naturelle, il ne reste que de retourner aux sources latines (v. Rainer 2003, à paraître a et b), ce qui d'ailleurs depuis quelque temps est grandement facilité par Google Books. Généralement, les termes français sont de simples calques du latin scientifique, mais parfois la situation s'avère plus compliquée : ainsi j'ai pu montrer (v. Rainer 2007) que le fr. mammifère, malgré son caractère latinisant, et contrairement à ce qu'en disent certains dictionnaires étymologiques, n'est pas un calque de mammifer, qui n'a jamais été en usage dans le latin scientifique, mais une formation latinisante française. Du point de vue de la formation des mots, il s'agit d'étudier quand et à quel point les différents types latins ont été intégrés dans le système de la formation des mots du français. C'est un autre vaste champ pratiquement vierge, dont l'étude est fondamentale pour améliorer le traitement lexicographique des mots savants mais aussi leur traitement synchronique dans la formation des mots.

\section{Conclusion}

Pour terminer, j'insisterai surtout encore une fois sur la situation privilégiée dont jouissent, en principe, les études diachroniques sur la formation des mots en français grâce à l'état excellent de la lexicographie historique et étymologique. À cela s'ajoute le privilège, que le français partage avec ses langues sœurs, d'avoir une langue mère bien documentée, le latin, et de pouvoir compter en plus, en France, avec la tradition la plus solide d'étude de la formation des mots du latin. Si l'on se décidait à exploiter à fond ces atouts, le français devrait tout naturellement occuper le rôle de leader dans l'étude diachronique de la formation des mots au sein de la linguistique mondiale.

\section{Références bibliographiques}

Amiot, D. / De Mulder, W. (2002). De l'adverbe au préfixe en passsant par la préposition : un phénomène de grammaticalisation?. Lingvistica Investigationes 25, 247-273.

Baldinger, K. (1972). Quelques problèmes de la suffixation dialectale. In : Straka, G. (ed.). Les dialectes de France au Moyen Âge et aujourd'hui. Paris : Klincksieck, 85-169.

Baldinger, K. (1995). Das Argotsuffix -zingue. In : Hoinkes, Ulrich (ed.). Panorama der Lexikalischen Semantik. Thematische Festschrift aus Anlaß des 60. Geburtstags von Horst Geckeler. Tübingen : Narr, 65-75.

Baldinger, K. (1997). Das Suffix -uche in der Volkssprache und im Argot. In : Holtus, G. et al. (éd.). Italica et Romanica. Festschrift für Max Pfister zum 65. Geburtstag. Tübingen : Niemeyer, vol. II, 115-147.

Blum, C. (ed.) (2001). Corpus de la littérature médiévale en langue d'oül des origines à la fin du XVe siècle. Prose narrative-Poésie - Théâtre. Paris : Champion électronique.

Bonnard, H. / Régnier, C. (1991). Petite grammaire de l'ancien français. Paris : Magnard.

Bork, H. D. (1968). Compte-rendu de Meyer-Lübke (1966). Romanische Forschungen 80, 421-439.

Bork, H. D. (1990). Die lateinisch-romanischen Zusammensetzungen Nomen + Verb und der Ursprung der romanischen Verb-Ergängzung-Komposita. Bonn : Romanistischer Verlag.

Büchi, E. (1996). Les structures du "Französisches Etymologisches Wörterbuch". Tübingen : Niemeyer. 
Buchwald, S. (1992). Französisches Subnormvokabular mit auslautendem -f. Ein Beitrag zur Wortbildung im Argot. Francfort : Lang.

Buridant, C. (1995). Les préverbes en ancien français. In : Rousseau, A. (ed.). Les préverbes dans les langues d'Europe. Villeneuve d'Ascq : Presses Universitaires du Septentrion, 287-323.

Buridant, C. (2000). Prolégomènes à une étude synthétique de la morphologie dérivationnelle en ancien français. L'information grammaticale 86, 14-20.

Cini, M. (ed.) (à paraître). I verbi sintagmatici in italiano e nelle varietà dialettali. Stato dell'arte e prospettive di ricerca. Francfort : Lang.

Corbin, D. / Corbin, P. (1991). Un traitement unifié du suffixe-ier(e). Lexique 10, 61-145.

Dufresne, M. / Dupuis, F. / Lontin, C.-M. (2001). Un changement dans la diachronie du français : la perte de la préfixation aspectuelle en a-. Revue québécoise de linguistique 29, 33-35.

Gather, A. (2001). Romanische Verb-Nomen-Komposita. Tübingen : Narr.

Höfler, M. (1967). Compte-rendu de Meyer-Lübke (1966). Zeitschrift für romanische Philologie 83, 104-114.

Hoven, R. (1994). Lexique de la prose latine de la Renaissance. Leiden : Brill.

Jänicke, O. (1969). Compte rendu de Meyer-Lübke (1966). Vox Romanica 28, 329-339.

Lachance, S. (1988). La condurrence suffixale en -eur (-euse) et -eux (-euse) en français québécois. Ottawa : Canadian Theses Service.

Lechanteur, J. (1999). Sur le suffixe -eresse en wallon et en Wallonie. Bulletin de la Commission Roayle de Toponymie et Dialectologie 71, 139-234.

Librova, Bohdana (à paraître). La particule séparable re- facteur de cohésion textuelle en français médiéval. In : Actes du XXIII Congrès International de Linguistique et de Philologie Romanes (Innsbruck 2007).

Lindemann, M. (1977). Zum Suffixwechsel von -eresse zu -euse und -trice im Französischen. Tübingen : Narr.

Lindner, T. (2003). Aspekte der lateinisch-romanischen Kompositaforschung. Moderne Sprachen 47, 115-141.

Löfstedt, B. (1994). Compte rendu de Bork (1990). Zeitschrift für romanische Philologie 110, 225-226.

Lüdtke, J. (2005). Romanische Wortbildung. Tübingen : Stauffenburg.

Martin, R. (2001). Le préfixe $a$-/ad- en moyen français. Romania 119, 289-322.

Martin, R. (2006). Sémantique préfixale du moyen français : les préfixes de-/des-. Lexique 17, 29-53.

Meyer-Lübke, W. ('21966 [1921]). Historische Grammatik der französischen Sprache. Zweiter Teil: Wortbildungslehre. Zweite, durchgesehene und ergänzte Auflage von J. M. Piel. Heidelberg : Winter.

Mongin, S. (1991). De quelques aspects de la dérivation dans les parlers ruraux de la Lorraine et de la Picardie. Verbum 14, 317-328.

Nyrop, K. (1908). Grammaire historique de la langue française. Tome troisième. Copenhagen : Nordisk Forlag.

Rainer, F. (2003a). Semantic fragmentation in word-formation: the case of Spanish -azo. In : Singh, R. / Starosta, S. (eds.). Explorations in Seamless Morphology. New Dehli etc. : Sage, 197-211.

Rainer, F. (2003b). L'intégration des composés latins du type aurifer en français. In : Fradin, B. et al. (eds.). Les unités morphologiques. Villeneuve d'Ascq : Silex, 151-168. (= Silexicales III)

Rainer, F. (2004). From Latin to French. In : Booij, G. et al. (éds.). Morphologie. Ein internationales Handbuch zur Flexion und Wortbildung. 2. Halbband / Morphology. A Handbook on Inflection and Word Formation. Volume 2. Berlin-New York : de Gruyter, 1698-1712.

Rainer, F. (2005a). Semantic change in word formation. Linguistics 43, 415-441.

Rainer, F. (2005b). Noms d'instruments/de lieux en -tor dans la Galloromania. Vox Romanica 64, 121-140.

Rainer, F. (2007). Frz. mammifère, oder: Habent sua fata et termini technici. Zeitschrift für französische Sprache und Literatur 117, 14-24. 
Rainer, F. (à paraître a). Neo- and Neo-Latin. Word Structure 1 (2008).

Rainer, F. (à paraître b). Die Integration des lateinischen Kompositionstyps tauriformis im Französischen und Italienischen. Zeitschrift für romanische Philologie 125 (2009).

Rézeau, P. (1997-98). Le suffixe -ouille en français de France. Travaux de linguistique et de philologie 35/36, 245362.

Roché, M. (2002). Aux origines du suffixe -ouille(r). In : Aurnague, M. / Roché, M. (eds.). Hommage à Jacques Allières. Vol. II : Romania sans frontières. Biarritz : Atlantica, 561-572.

Roché, M. (2006). La dérivation en -ier(e) en ancien français. Lexique 17, 55-96.

Roché, M. (2008). Quelques exemples de morphologie non conventionnelle dans les formations construites à partir d'un mot en -ouille(r). In : Fradin, B. (éd.). La raison morphologique. Hommge à la mémoire de Danielle Corbin. Amsterdam : Benjamins, 215-238.

Schpak-Dolt, N. (2003). Bibliographische Materialien zur französischen Morphologie. Francfort : Lang.

Thibault, A. (1989). La terminaison lat. -uus dans les emprunts savants en français. Revue de linguistique romane 53, 85-110.

Turcan, I. (2001). Mémoire de préverbes passés : usages linguistiques et littéraires, et témoignage de quelques dictionnaires de langue française jusqu'au XIX ${ }^{\mathrm{e}}$ siècle. L'information grammaticale 90, 50-61.

\footnotetext{
${ }^{1}$ Un panorama, forcément très succinct, de l'histoire de la formation des mots en français est fourni aussi dans Rainer (2004 : 1704-1710). Dans une perspective romane, on peut consulter avec profit Lüdtke (2005).

${ }^{2}$ V. pourtant Bonnard / Régnier (1991), qui contient une liste de suffixes nominaux.

${ }^{3}$ Pour s'y retrouver plus facilement, v. Büchi (1996), ainsi que l'index en deux volumes (Paris : Champion 2003).

${ }^{4}$ Disponible sur internet sous: http://www.ucalgary.ca/ dewalker/Dictionary/dict.html.

${ }^{5}$ V. http://atilf.atilf.fr/dmf.htm.

${ }^{6} \mathrm{~V} . \mathrm{http}: / /$ zeus.inalf.fr/tlf.htm.

${ }^{7}$ V. http://www.atilf.fr/tlf\%2Detym/.

${ }^{8}$ V.http://www.frantext.fr/frtcategpass.htm.

${ }^{9}$ Seulement pour l'histoire du vocabulaire scientifique l'Oxford English Dictionary (Oxford: Clarendon 1991; pour la troisième édition en cours v. http://www.oed.com/) reste supérieur.

${ }^{10}$ Malheureusement, il existe très peu de travaux d'ensemble sur des dialectes spécifiques (cf., p.ex., Mongin 1991), ce serait là un autre vaste champ à travailler par les morphologues.

${ }^{11}$ Sur le préfixe $a$-/ad-, v. Dufresne et al. (2001) et Martin (2001). Sur re-, séparable en ancien français, Librova (à paraître).

${ }^{12}$ Le phénomène de la grammaticalisation et la frontière entre syntaxe, composition et préfixation se trouvent aussi au centre d'une série de travaux récents de D. Amiot (v., p. ex., Amiot / De Mulder 2002).
} 